\title{
National trends in the proportion of in- hospital deaths by cause of death among older adults with long-term care: a nationwide observational study in Japan from 2007 to 2017
}

\author{
Yuta Taniguchi 1,2, Masao Iwagami ${ }^{2,3^{*}}$, Xueying Jin ${ }^{2,3}$, Nobuo Sakata ${ }^{2,3}$, Mikiya Sato ${ }^{2,3,4}$, Taeko Watanabe ${ }^{2}$, \\ Kyoko Hanari ${ }^{2}$, Kazuhiro Abe ${ }^{5,6}$, Haruko Noguchi $^{2,3,7}$ and Nanako Tamiya ${ }^{2,3}$
}

\begin{abstract}
Background: Japan has promoted end-of-life care at home and in long-term care facilities, and the total proportion of in-hospital deaths has decreased recently. However, the difference in trends of in-hospital deaths by the cause of death remains unclear. We investigated the variation in trends of in-hospital deaths among older adults with longterm care from 2007 to 2017, by cause of death and place of care.

Methods: Using the national long-term care insurance registry, long-term care claims data, and national death records, we identified people aged 65 years or older who died between 2007 and 2017 and used long-term care services in the month before death. Using a joinpoint regression model, we evaluated time trends of the proportion of in-hospital deaths by cause of death (cancer, heart diseases, cerebrovascular diseases, pneumonia, and senility) and place of care (home, long-term care health facility, or long-term care welfare facility).

Results: Of the 3,261,839 participants, the mean age was $87.0 \pm 8.0$ years, and $59.2 \%$ were female. Overall, the proportion of in-hospital deaths decreased from $66.2 \%$ in 2007 to 55.3\% in 2017. By cause of death, the proportion of inhospital deaths remained the highest for pneumonia ( $81.6 \%$ in 2007 and $77.2 \%$ in 2017) and lowest for senility (25.5\% in 2007 and $20.0 \%$ in 2017) in all types of places of care. The joinpoint regression analysis showed the steepest decline among those who died of senility, especially among long-term care health facility residents.

Conclusions: The findings of this nationwide study suggest that there was a decreasing trend of in-hospital deaths among older adults, although the speed of decline and absolute values varied widely depending on the cause of death and place of care.
\end{abstract}

Keywords: End-of-life care, Place of death, Health services research, Japan, Long-term care

*Correspondence: iwagami-tky@umin.ac.jp

${ }^{3}$ Department of Health Services Research, Faculty of Medicine, University

of Tsukuba, 1-1-1 Tenno-dai, Tsukuba, Ibaraki 305-8575, Japan

Full list of author information is available at the end of the article

\section{Background}

There is a rise in the proportion of older adult populations worldwide due to decline in fertility rates and extension of life expectancy [1]. This makes where to provide end-of-life care a matter of global interest. Japan is facing a rapid aging of the population and has the highest proportion of older adults in the world [1]. The Japanese government introduced a long-term care insurance 
system in 2000 to cover the costs for long-term care of people aged 65 or older, and people aged 40-64 with specific diseases [2].

As the population is aging, the number of deaths is also increasing. In 2017, 73\% of the deaths in Japan occurred in hospitals [3], which was higher than that in other countries such as the United States (29.8\%), England (46.0\%), or Canada (59.9\%) [4]. Meanwhile, 55\% of the general population in Japan hope to die at home [5]. Also, the number of deaths in Japan is expected to increase from 1.3 million in 2017 to 1.6 million by 2030 [3, 6], and hospital bed capacity as a place of death is predicted to be in short supply [7, 8]. To fill this gap, the Japanese government has, since 2006, promoted end-of-life care at home and at long-term care (LTC) facilities, by increasing the financial incentives for service providers who attend to patients' deaths at home and at LTC facilities [9-11].

Overall, it has been reported that after peaking at $79.8 \%$ in 2005 , the proportion of in-hospital deaths has decreased gradually to $73.0 \%$ in 2017 [3]. Moreover, a previous study revealed a decreasing trend of in-hospital deaths among older adults using LTC services, with a sharper reduction among those in a residential aged care facility than in the community, possibly due to the incentivization of end-of-life care outside hospitals [12]. A few other studies on LTC welfare facility residents [13] and patients with dementia [14] also speculated that the declining trends of in-hospital deaths may be attributable to the promotion of end-of-life care at home and in LTC facilities. However, these previous studies did not evaluate differences in trends of in-hospital deaths between the causes of death. To promote end-of-life care at home and in LTC facilities, more detailed information would be helpful for planning cause-and-place-specific strategies, as the use and needs of services can differ between diseases. Therefore, this descriptive study aimed to evaluate the difference in transition of in-hospital deaths among older adults in LTC between 2007 and 2017, by cause of death in different types of places of care.

\section{Methods}

\section{Data source}

We obtained data for the period 2007 to 2017, from the national long-term care insurance (LTCI) registry, LTCI claims data and national death records, with permission from the Ministry of Health, Labour, and Welfare under the Statistics Act, Article 33. First, we identified older adults ( $\geq 65$ years) who were withdrawn from the LTCI registry and linked them to national death records, using the following four variables: insurer code (city code), sex, birth year and month, and disqualification date (i.e., moving out or death). We excluded individuals who were not uniquely identifiable with these four variables. Second, using LTCI claims data, we identified individuals who used the LTCI services in the month before death, with their place of care (i.e., home, LTC health facilities, or LTC welfare facilities).

Using the 10th revision of the International Statistical Classification of Diseases (ICD-10) codes, we identified individuals who died of the following major causes of death in Japan [3]: cancer (C00-C97), heart diseases (I01I02, I05-I09, I20-I25, I27, I30-I51), cerebrovascular diseases (I60-I69), pneumonia (J12-J18), and senility (R54).

\section{Study population}

The inclusion criteria of the study were i) individuals aged 65 or older, ii) those who died between 2007 and 2017, iii) those with care need level 1 or above, and iv) those who used LTC services at home or LTC facilities in the month before death. Under the LTC insurance system in Japan, eligibility for LTC services is assessed and categorized into seven levels: support level 1 (least disabled), 2 and care need levels 1-5 (most disabled) [2]. We included persons with care need level 1 or above because individuals with support levels 1-2 have limited eligibility for LTC facility services $[15,16]$. We categorized the study population into three groups according to the place of care in the month before death: home, LTC health facilities (also called geriatric health services facilities, or roujinhokenshisetsu or "roken" in Japanese), and LTC welfare facilities (also called special nursing homes, or tokubetsuyougoroujinhoumu or "tokuyo" in Japanese). The LTC health facility is a rehabilitation facility that is used for short periods and employs a full-time physician [9]. The LTC welfare facility is a daily life facility providing constant care services, where a part-time physician is permissible [9]. Also, since 2006, both the LTC health facilities and welfare facilities have been encouraged to provide end-of-life care by financial incentives from the Japanese government [9-11].

The exclusion criteria were i) those living in group homes for older adults with dementia and geriatric apartments and ii) few patients who lived at multiple types of sites in the month before death. Group homes for older adults with dementia and geriatric apartments provide residents with services such as housework help or longterm care, and their characteristics as places of care are different from one's own home [9].

\section{Statistical analysis}

We calculated the proportion of in-hospital deaths for each year from 2007 to 2017, as the number of people who died in a hospital divided by the number of total deaths, overall (any hospital death) and by the cause of death (cancer, heart diseases, cerebrovascular diseases, 
pneumonia, and senility) in each place of care (home, LTC health facility, or LTC welfare facility).

To evaluate the time trends of the proportion of inhospital deaths, we fitted the joinpoint regression model for each cause of death in each place of care. In the joinpoint regression model, we determined the trend change points (joinpoint), estimated the annual percentage change between joinpoints, and calculated the average annual percentage change [17]. To investigate the trend difference by care need levels, we repeated the analysis by care need levels (care need level 1-2 [low to moderate need] or care need level 3-5 [moderate to severe need]). $P$-values $<0.05$ were considered statistically significant.

We used Stata 15 (StataCorp, TX, USA), Microsoft Excel for Mac 16.33 (Microsoft, WA, USA), and Joinpoint Regression Program 4.9.0.0 (National Cancer Institute, MD, USA) for analysis.

\section{Additional analysis}

Since the age distribution may have changed over the decade, we conducted additional analyses using a logistic regression model adjusting for age. We calculated the adjusted odds ratios of in-hospital death for each cause of death at each place of care in each year from 2008 to 2017 (reference: 2007).

\section{Ethics approval}

Because of the anonymized nature of the data, individual participants' consent was waived. The study was approved by the Ethics Committee of the University of Tsukuba (approval no. 1324-2).

\section{Results}

From the 6,632,539 older adults who were eligible from the LTCI registry during the period between 2007 and 2017, we uniquely identified 6,426,937 people (96.9\%) using the four variables (insurer city code, sex, birth year and month, and disqualification date), and successfully linked 5,722,084 people (86.3\%) with the national death records. Of this population, 3,261,839 utilized LTC services in the month before death with care need level 1 or higher. These included 1,947,489 people at home, 383,522 at the LTC health facility, and 588,838 at the LTC welfare facility. The 24,079 patients who received LTCI services at multiple sites (home, LTC health facility, or LTC welfare facility) in the month before death were excluded in the analysis. The number of final study participants was $2,919,849$. Table 1 shows the characteristics of the participants. The mean age at death was $87.0 \pm 8.0$ years and $59.2 \%$ were female.

The overall proportion of in-hospital deaths decreased from $66.2 \%$ in 2007 to $55.3 \%$ in 2017. Between 2007 and 2017, the percentage of in-hospital deaths declined, by cause of death, from 70.0 to $59.4 \%$ for cancer $(n=586,002)$, from 64.3 to $60.1 \%$ for heart diseases ( $n=491,054)$, from 61.5 to $51.2 \%$ for cerebrovascular diseases $(n=290,470)$, from 81.6 to $77.2 \%$ for pneumonia $(n=345,316)$, and from 25.5 to $20.0 \%$ for senility $(n=320,517)$.

The trends of in-hospital deaths by cause of death and place of care are presented in Figs. 1, 2, 3. The proportion of in-hospital deaths remained the highest for pneumonia and lowest for senility in all three types of places of care. The joinpoint regression analysis showed that the average annual percentage change was statistically significant for all causes of death in all types of places of care, except for the LTC welfare facility residents who died of pneumonia (Table 2). The declining trend for each cause of death was the sharpest among the LTC health facility residents, followed by the LTC welfare facility residents or those residing at home. The average annual percentage decrease was largest for the LTC health facility residents who died of senility (6.1\%).

The results of the analyses by care need levels are shown in the additional files (Additional Figs. 1-2 and Additional Table 1). The results were similar, and the proportion of in-hospital deaths remained the highest for pneumonia and lowest for senility regardless of the care need level. People with higher care need levels showed a steeper decline in in-hospital deaths for every cause of death. The additional analyses with the logistic regression models adjusting for age showed similar trends (Additional Figs. 3, 4, 5).

\section{Discussion}

This study, to the best of our knowledge, is the first to investigate the difference in the transition of the proportion of in-hospital deaths among older adults in LTC by cause of death and place of care. From 2007 to 2017, there has been a decreasing trend in in-hospital deaths among older adults regardless of the cause of death. The speed of the decline and absolute values varied widely between the cause of death and place of care, possibly due to the different prognosis for each disease.

By cause of death, the proportion of in-hospital deaths remained highest for pneumonia throughout the study period and lowest for senility in all three types of places of care. A questionnaire survey in 241 LTC welfare facilities reported that not having pneumonia as the cause of death was positively associated with dying at LTC welfare facilities [13]. Our study showed comparable results at the national level, not only for the LTC welfare facility but also for the LTC health facility and at home. We assume that patients with pneumonia have a higher possibility of recovery than those with other conditions such as senility, and this may have led to their transfer to hospital 
Table 1 Characteristics of older adults who used long-term care services in the month before death

\begin{tabular}{|c|c|c|c|}
\hline & \multicolumn{3}{|c|}{ Place of care in the month before death } \\
\hline & Home & LTC health facility & LTC welfare facility \\
\hline No & $1,947,489$ & 383,522 & 588,838 \\
\hline Age, years, Mean (SD) & $85.8(8.1)$ & $88.9(7.2)$ & $89.8(7.2)$ \\
\hline Female sex, No (\%) & $1,039,644(53.4)$ & $251,900(65.7)$ & $436,990(74.2)$ \\
\hline \multicolumn{4}{|l|}{ Cause of death, No (\%) } \\
\hline Cancer & $507,275(26.0)$ & $37,179(9.7)$ & $41,548(7.1)$ \\
\hline Heart diseases & $332,048(17.1)$ & $67,630(17.6)$ & $91,376(15.5)$ \\
\hline Cerebrovascular diseases & $157,555(8.1)$ & $53,267(13.9)$ & $79,648(13.5)$ \\
\hline Pneumonia & $198,867(10.2)$ & $59,566(15.5)$ & $86,883(14.8)$ \\
\hline Senility & $154,420(7.9)$ & $51,149(13.3)$ & $114,948(19.5)$ \\
\hline The others & $597,324(30.7)$ & $114,731(29.9)$ & $174,435(29.6)$ \\
\hline \multicolumn{4}{|l|}{ Year of death } \\
\hline 2007 & 127,741 & 26,003 & 38,081 \\
\hline 2008 & 140,520 & 27,991 & 42,630 \\
\hline 2009 & 142,469 & 28,517 & 43,404 \\
\hline 2010 & 154,507 & 30,801 & 46,317 \\
\hline 2011 & 166,153 & 33,214 & 50,407 \\
\hline 2012 & 182,448 & 36,049 & 54,907 \\
\hline 2013 & 185,934 & 38,022 & 57,883 \\
\hline 2014 & 195,295 & 38,875 & 58,942 \\
\hline 2015 & 218,885 & 38,169 & 58,588 \\
\hline 2016 & 211,707 & 42,313 & 66,462 \\
\hline 2017 & 221,830 & 43,568 & 71,217 \\
\hline
\end{tabular}

LTC long-term care, SD standard deviation

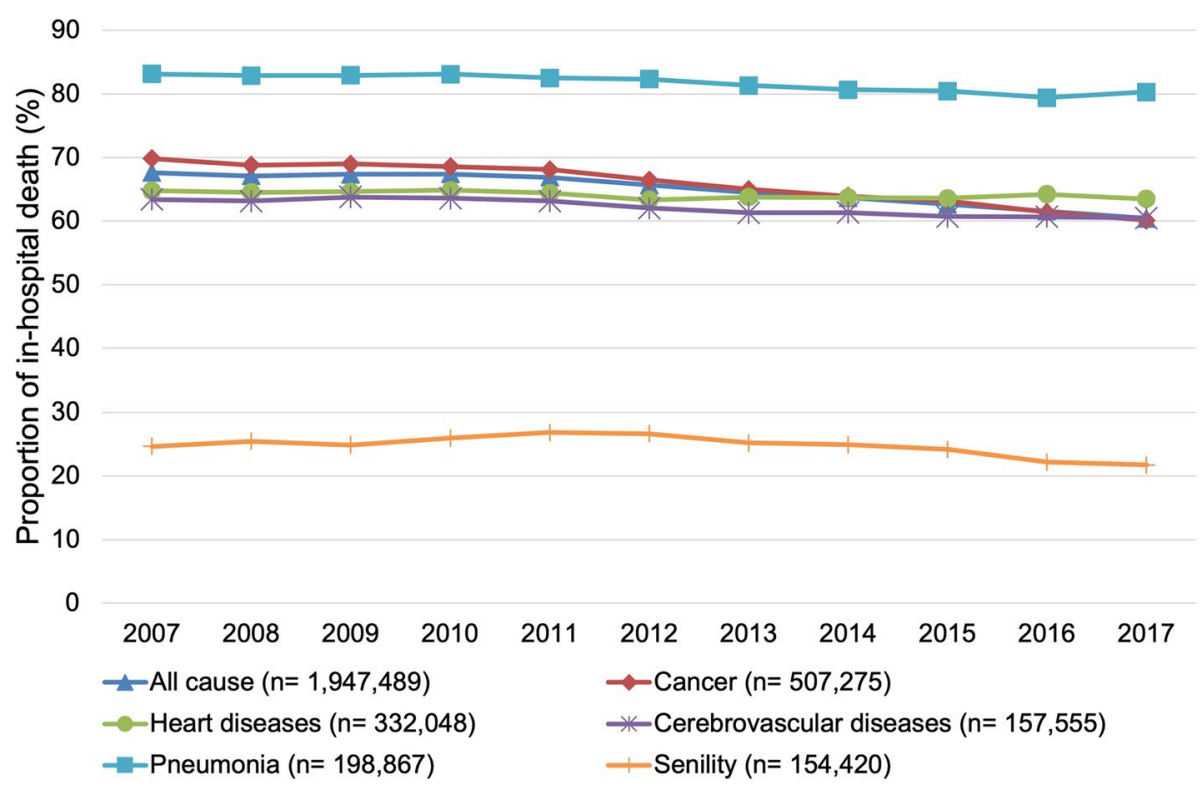

Fig. 1 Trends of the proportion of in-hospital deaths of those living at home in the month before death $(n=1,947,489)$ 


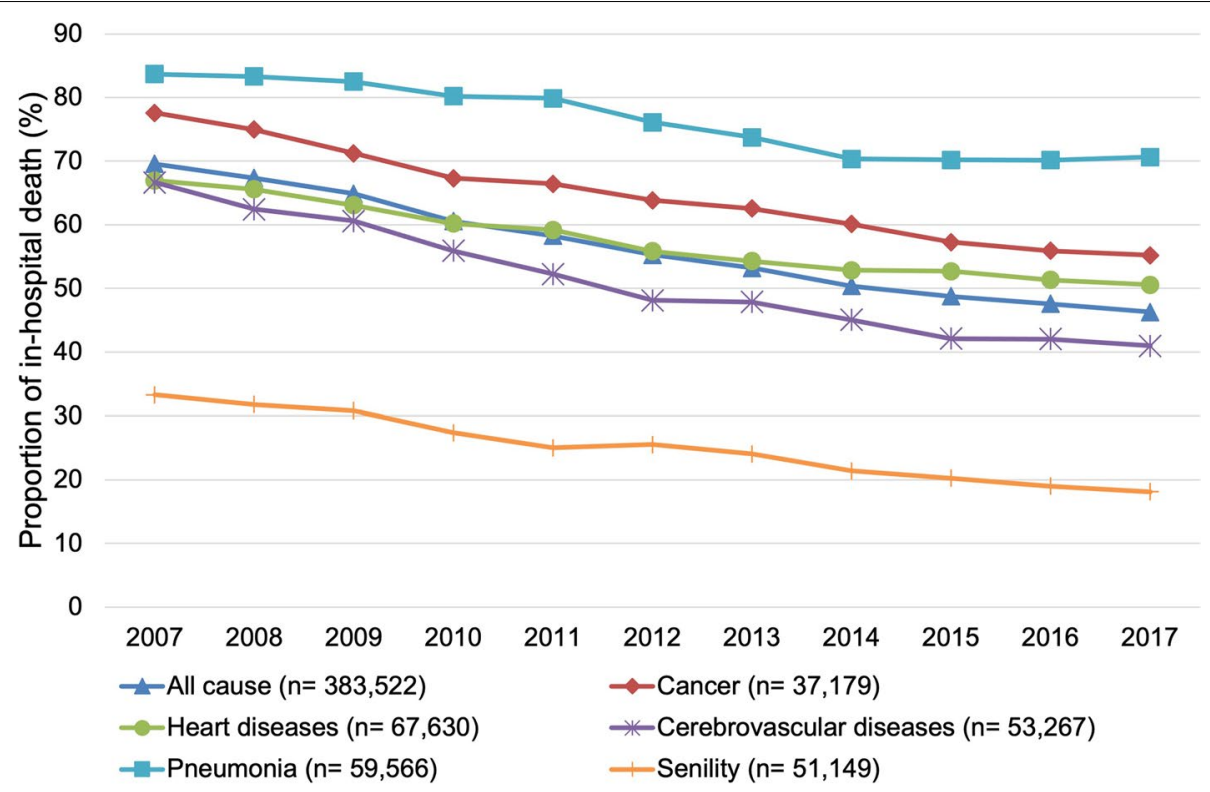

Fig. 2 Trends of the proportion of in-hospital deaths of long-term care health facility residents in the month before death $(n=383,522)$

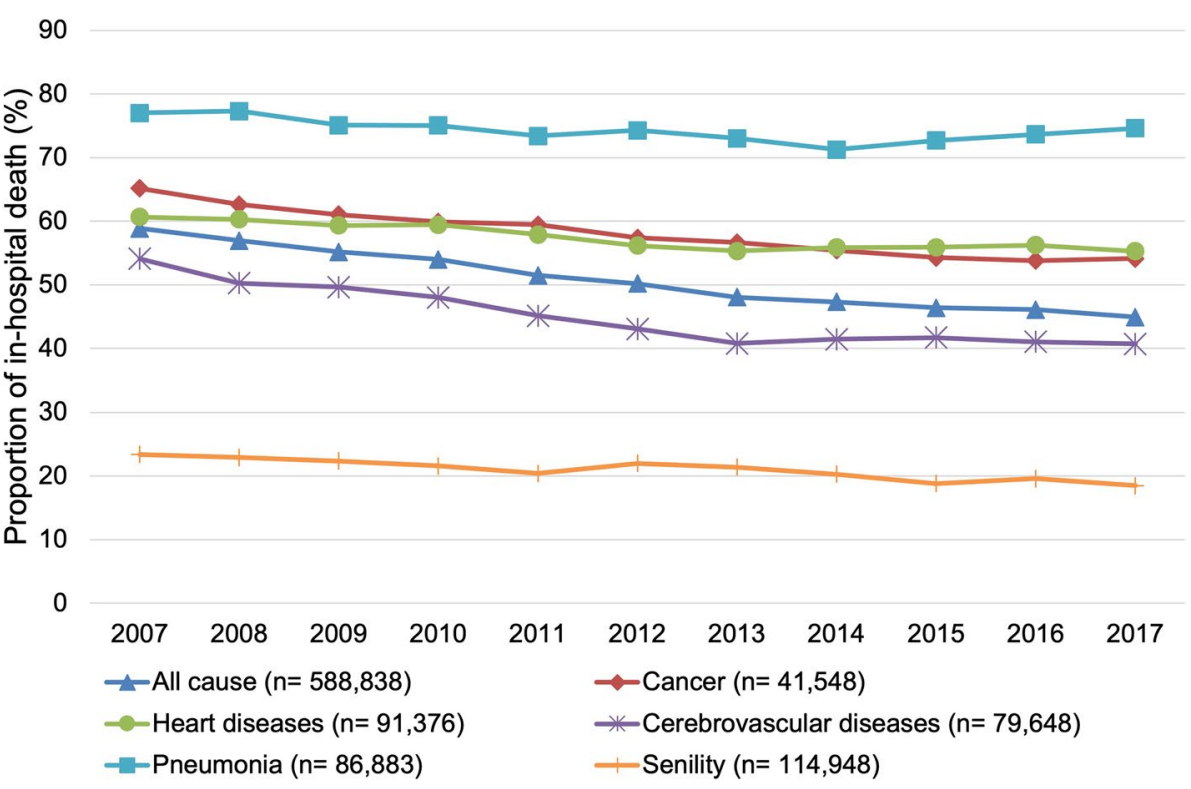

Fig. 3 Trends of the proportion of in-hospital deaths of long-term care welfare facility residents in the month before death $(n=588,838)$

even at the terminal stage, and consequently in-hospital deaths. Conversely, the decreasing trend of in-hospital deaths was sharpest among patients with senilty, especially among LTC health facility residents. This may suggest an improvement in end-of-life care among patients with senility, especially at LTC health facilities.

Not only for senility but also for every cause of death, we found the steepest decline in in-hospital deaths among LTC health facility residents, followed by LTC welfare facility residents or those living at home. This finding is consistent with that of a previous study that reported a sharper reduction in in-hospital deaths among those at the LTC facilities than in the community [12]. However, our study further differentiated LTC health facility and LTC welfare facility to reveal more details, in that the declining trend was steeper among LTC health facility residents than among LTC welfare facilities. This variation may have been caused by the difference in 
Table 2 Trends of the proportion of in-hospital deaths, by cause of death and place of care

\begin{tabular}{|c|c|c|c|c|c|c|c|}
\hline & & Trend1 & & Trend2 & & & \\
\hline Place of care & Cause of death & Years & APC (\%) & Years & APC (\%) & Average APC & {$[95 \% \mathrm{Cl}]$} \\
\hline \multirow[t]{6}{*}{ Home } & All cause & $2007-2011$ & -0.2 & $2011-2017$ & $-1.7^{*}$ & $-1.1^{*}$ & {$[-1.2$ to -1.0$]$} \\
\hline & Cancer & $2007-2011$ & $-0.6^{*}$ & 2011-2017 & $-2.0^{*}$ & $-1.4^{*}$ & {$[-1.6$ to -1.2$]$} \\
\hline & Heart diseases & 2007-2017 & $-0.2^{*}$ & & & $-0.2^{*}$ & {$[-0.3$ to -0.1$]$} \\
\hline & Cerebrovascular diseases & $2007-2017$ & $-0.6^{*}$ & & & $-0.6^{*}$ & {$[-0.8$ to -0.4$]$} \\
\hline & Pneumonia & $2007-2017$ & $-0.5^{*}$ & & & $-0.5^{*}$ & {$[-0.6$ to -0.3$]$} \\
\hline & Senility & 2007-2012 & $1.6^{*}$ & $2012-2017$ & $-4.1^{*}$ & $-1.3^{*}$ & {$[-2.2$ to -0.4$]$} \\
\hline \multirow[t]{6}{*}{ LTC health facility } & All cause & 2007-2014 & $-4.6^{*}$ & 2014-2017 & $-2.9^{*}$ & $-4.1^{*}$ & {$[-4.5$ to -3.7$]$} \\
\hline & Cancer & 2007-2017 & $-3.4^{*}$ & & & $-3.4^{*}$ & {$[-3.7$ to -3.1$]$} \\
\hline & Heart diseases & 2007-2013 & $-3.6^{*}$ & 2013-2017 & $-1.8^{*}$ & $-2.9^{*}$ & {$[-3.3$ to -2.4$]$} \\
\hline & Cerebrovascular diseases & 2007-2015 & $-5.5^{*}$ & 2015-2017 & -1.1 & $-4.7^{*}$ & {$[-5.8$ to -3.5$]$} \\
\hline & Pneumonia & 2007-2017 & $-2.1^{*}$ & & & $-2.1^{*}$ & {$[-2.6$ to -1.7$]$} \\
\hline & Senility & $2007-2017$ & $-6.1^{*}$ & & & $-6.1^{*}$ & {$[-6.6$ to -5.5$]$} \\
\hline \multirow[t]{6}{*}{ LTC welfare facility } & All cause & 2007-2013 & $-3.3^{*}$ & 2013-2017 & $-1.7^{*}$ & $-2.7^{*}$ & {$[-2.9$ to -2.4$]$} \\
\hline & Cancer & 2007-2015 & $-2.1^{*}$ & 2015-2017 & -0.1 & $-1.7^{*}$ & {$[-2.2$ to -1.2$]$} \\
\hline & Heart diseases & $2007-2013$ & $-1.5^{*}$ & 2013-2017 & -0.0 & $-0.9^{*}$ & {$[-1.4$ to -0.5$]$} \\
\hline & Cerebrovascular diseases & 2007-2013 & $-4.3^{*}$ & 2013-2017 & -0.2 & $-2.7^{*}$ & {$[-3.3$ to -2.1$]$} \\
\hline & Pneumonia & $2007-2014$ & $-1.1^{*}$ & 2014-2017 & 1.3 & -0.4 & {$[-0.8$ to 0.1$]$} \\
\hline & Senility & $2007-2017$ & $-2.2^{*}$ & & & $-2.2^{*}$ & {$[-2.8$ to -1.5$]$} \\
\hline
\end{tabular}

* Significantly different from zero $(p<0.05)$. LTC long-term care, APC annual percent change

accessible medical care in each place of care. Attending death would be more feasible at the LTC health facility, which employs a full-time physician, than an LTC welfare facility, where often only a part-time physician is available. In addition, the Japanese government added payment for the doctor's visiting the LTC health facilities at night and on holidays since 2008 [18], which may have encouraged medical treatment at the LTC health facility. Additionally, we considered the possible influence of financial incentives for LTC health facilities treating pneumonia which were introduced in 2012, with the aim of reducing the transfer of patients with pneumonia to the hospital [19]. However, we did not observe a statistically significant change in the decreasing trend before and after the introduction of the incentive in 2012, among those who died of pneumonia at the LTC health facility.

Finally, by care need levels, we observed a steeper decreasing trend in in-hospital deaths among those with higher care need levels. This is consistent with findings of previous studies which indicated that a higher care need level or a poorer functional status was negatively associated with in-hospital deaths $[12,20]$. As the level of care needs deteriorated, the five-year survival rates showed a decline, from $72.3 \%$ for people with the least disability support level, to $22.2 \%$ for those with care need level 5 (most disabled) [21]. The most common occasion for health professionals to confirm patients' intentions about their desired medical care near death, was when patients were close to death [22]. Therefore, older adults with higher care need levels, who had a shorter prognosis, may have had more chance to make their desire for end-of-life care known, which could have resulted in the fewer inhospital deaths. Promotion of patients' self-determination at the end of life in the recent years by the Japanese government [23], might have led to steeper decreasing trends in in-hospital deaths, especially among those with higher care need levels.

This study has several limitations. First, we could not confirm a causal relationship between the health policy to promote end-of-life care at home or LTC facilities and the declining trends of in-hospital death. Second, we were unable to consider underlying diseases other than the cause of death due to lack of information. In addition, the new coding rule of the underlying cause of death was implemented in 2017 according to the update of ICD-10 in 2013 [24]. Therefore, the patient's disease condition and the cause of death may have been different prior to and after 2017. Third, we could not link the LTCI registry to national death records for a few participants who were not uniquely identified with available variables. Finally, while we have discussed the differences between available medical resources among the three types of places of care that may explain the variation in the proportion of inhospital deaths, we did not consider the individual characteristics of each patient or LTC facility; these might have affected the proportion of in-hospital deaths. 


\section{Conclusions}

In conclusion, among older adults who lived at home or in a LTC facility in the month before death, the proportion of in-hospital deaths decreased between 2007 and 2017, and it remained the highest for pneumonia and the lowest for senility. The decreasing trend was observed to be greater in senile patients, especially among the LTC health facility residents, which may suggest an improvement in end-of-life care at the LTC health facilities. To expand the end-of-life care at home and at LTC facilities, the assessment and enhancement of care appropriate for each disease could be effective. Moreover, since Japan has the highest proportion of older people globally, our results could be informative for the other countries experiencing an increasingly aged population.

\section{Abbreviations}

ICD-10: International Classification of Diseases, Tenth Revision; LTC: Long-term care; LTCI: Long-term Care Insurance.

\section{Supplementary Information}

The online version contains supplementary material available at https://doi. org/10.1186/s12877-021-02700-1.

Additional file 1: Additional Figure 1. Trends of the proportion of inhospital deaths of those in care need levels 1-2 in the month before death ( $n=729,847$ ). Additional Figure 2. Trends of the proportion of in-hospital deaths of those in care need levels 3-5 in the month before death $(n=$ 2,190,002). Additional Figure 3. Age-adjusted odds ratios for in-hospital deaths of those living at home in the month before death $(n=1,947,489)$. Additional Figure 4. Age-adjusted odds ratios for in-hospital deaths of long-term care health facility residents in the month before death $(n=$ 383,522). Additional Figure 5. Age-adjusted odds ratios for in-hospital deaths of long-term care welfare facility residents in the month before death $(n=588,838)$

Additional file 2: Additional Table 1. Trends of the proportion of inhospital deaths, by cause of death and care need levels.

\section{Acknowledgements}

We would like to thank Editage (www.editage.com) for its English language editing.

\section{Authors' contributions}

Y.T. planned the study, performed all data analysis, and wrote the paper. M.I., M.S., and N.T. supervised the study and helped revise the manuscript. X.J., T.W., and K.A. prepared the data for analysis and helped revise the manuscript. N.S., K.H., and H.N. designed the study and reviewed the paper. The authors read and approved the final manuscript.

\section{Funding}

This research was supported by a grant-in-aid from the Ministry of Health, Labour and Welfare; Health and Labour Sciences Research Grant, Japan; (H30choju-ippan-007). The funder had no role in the interpretation of the data.

\section{Availability of data and materials}

The datasets generated and/or analyzed during the current study are not publicly available because the Japanese Ministry of Health, Labour and Welfare owns the original data and only approved the secondary use of the data for the current study.

\section{Declarations}

Ethics approval and consent to participate

The study was approved by the Ethics Committee of the University of Tsukuba (approval no. 1324-2). All methods were performed in accordance with the relevant guidelines and regulations. Consent to participate was waived as we used anonymized claims data and national death records.

\section{Consent for publication}

Not applicable.

\section{Competing interests}

The authors declare no competing interests.

\section{Author details}

${ }^{1}$ Graduate School of Comprehensive Human Sciences, University of Tsukuba, Ibaraki, Japan. ${ }^{2}$ Health Services Research and Development Center, University of Tsukuba, Ibaraki, Japan. ${ }^{3}$ Department of Health Services Research, Faculty of Medicine, University of Tsukuba, 1-1-1 Tenno-dai, Tsukuba, Ibaraki 305-8575, Japan. ${ }^{4}$ Sumitomo Heavy Industries, Ltd., Human Resources Group, Health Services Center, Tokyo, Japan. ${ }^{5}$ Department of Public Health, Graduate School of Medicine, The University of Tokyo, Tokyo, Japan. ${ }^{6}$ Takemi Program in International Health, Harvard T.H.Chan School of Public Health, Boston, MA, USA.

${ }^{7}$ Faculty of Political Science and Economics, Waseda University, Tokyo, Japan.

Received: 12 July 2021 Accepted: 1 December 2021

Published online: 03 January 2022

\section{References}

1. World Health Organization. World report on ageing and health. 2015. https://apps.who.int/iris/handle/10665/186463. Accessed 14 Oct 2021.

2. Iwagami M, Tamiya N. The long-term care insurance system in Japan: past, present, and future. JMA J. 2019;2(1):67-9.

3. Ministry of Health, Labour and Welfare, Japan. Vital statistics of Japan. 2019. https://www.mhlw.go.jp/english/database/db-hw/index.html. Accessed 14 Oct 2021.

4. Cross SH, Warraich HJ. Changes in the place of death in the United States. N Engl J Med. 2019;381(24):2369-70.

5. Cabinet Office Japan. Koureisha no kenko ni kansuru ishikichosakekka. 2012. https://www8.cao.go.jp/kourei/ishiki/h24/sougou/zentai/index. html. Accessed 14 Oct 2021.

6. National Institute of Population and Social Security Research. Population projections for Japan. 2017. http://www.ipss.go.jp/pp-zenkoku/j/zenko ku2017/pp_zenkoku2017.asp. Accessed 14 Oct 2021. In Japanese.

7. Ministry of Health, Labour and Welfare, Japan. Shinryohoshu to kaigohoshu no doujikaitei ni mukete (shiryo 1). 2011. https://www.mhlw.go.jp/ stf/shingi/2r9852000001 rbxs.html. Accessed 14 Oct 2021. In Japanese.

8. Ohta H. Chiiki houkatsu keashisutemu (community-based integrated care system). Nakayama Shoten. 2016;1:245. In Japanese.

9. Sugimoto K, Ogata Y, Kashiwagi M. Factors promoting resident deaths at aged care facilities in Japan: a review. Health Soc Care Community. 2018:26(2):e207-24.

10. Ministry of Health, Labour and Welfare, Japan. Zaitakuiryo (sono 1). 2019. https://www.mhlw.go.jp/stf/shingi2/0000212500_00045.html. Accessed 14 Oct 2021. In Japanese.

11. Ikegami N, Ikezaki S. Nursing homes and end-of-life care in Japan. J Am Med Dir Assoc. 2013;14(10):718-23.

12. Sato M, Tamiya N, Jin X, Watanabe T, Takahashi H, Noguchi H. Impact of a Japanese incentivization program to increase end-of-life care outside of hospitals. J Am Med Dir Assoc. 2021;22(2):329-33.

13. Ikegami $N$, Ikezaki S. Japan's policy of promoting end-of-life care in nursing homes: impact on facility and resident characteristics associated with the site of death. Health Policy. 2012;105(2-3):303-11.

14. Koyama T, Sasaki M, Hagiya H, et al. Place of death trends among patients with dementia in Japan: a population-based observational study. Sci Rep. 2019;9(1):20235. 
15. Tsutsui T, Muramatsu N. Japan's universal long-term care system reform of 2005: containing costs and realizing a vision. J Am Geriatr Soc. 2007;55(9):1458-63.

16. Morikawa M. Towards community-based integrated care: trends and issues in Japan's long-term care policy. Int J Integr Care. 2014;14:e005.

17. Kim HJ, Fay MP, Feuer EJ, Midthune DN. Permutation tests for joinpoint regression with applications to cancer rates. Stat Med. 2000;19(3):335-51.

18. Ministry of Health, Labour and Welfare, Japan. Kaigo service riyosha nitaisuru iryo teikyo no arikata nitsuite (shiryo 1). 2011. https://www. mhlw.go.jp/stf/shingi/2r9852000001nv62.html. Accessed 14 Oct 2021. In Japanese.

19. Ministry of Health, Labour and Welfare, Japan. Kaigoroujinhokenshisetsu no houshu, kijun ni tsuite (shiryo 2). 2017. https://www.mhlw.go.jp/stf/ shingi2/0000185798.html. Accessed 14 Oct 2021. In Japanese.

20. Honinx E, Piers RD, Onwuteaka-Philipsen BD, et al. Hospitalisation in the last month of life and in-hospital death of nursing home residents: a cross-sectional analysis of six European countries. BMJ Open. 2021;11(8):e047086.

21. Nagata H, Harada Y, Azemoto C, Wakui Y. Yokaigodo no keinenhenka -doitsushudan niokeru yokaigodobunpu no 9nenkan no henka. J Health Welf Stat. 2011;58:37-43 In Japanese.

22. Ministry of Health, Labour and Welfare, Japan. Heisei 29 nendo jinsei no saishudankai niokeru iryo ni kansuru ishikichosa. 2018. https://www. mhlw.go.jp/toukei/list/saisyuiryo_a.html. Accessed 14 Oct 2021. In Japanese.

23. Inoue M, Hanari K, Hamano J, Gallagher J, Tamiya N. Current engagement in advance care planning in Japan and its associated factors. Gerontol Geriatr Med. 2019;5:2333721419892694.

24. Ministry of Health, Labour and Welfare, Japan. Shippei, shogai oyobi shiin no toukeibunrui. https://www.mhlw.go.jp/toukei/sippei/. Accessed 14 Oct 2021. In Japanese.

\section{Publisher's Note}

Springer Nature remains neutral with regard to jurisdictional claims in published maps and institutional affiliations.

- fast, convenient online submission

- thorough peer review by experienced researchers in your field

- rapid publication on acceptance

- support for research data, including large and complex data types

- gold Open Access which fosters wider collaboration and increased citations

- maximum visibility for your research: over $100 \mathrm{M}$ website views per year

At BMC, research is always in progress.

Learn more biomedcentral.com/submissions 